\title{
Curb your fatty acids
}

\section{By Benjamin Boettner, Associate Editor}

A University of California, San Francisco team has uncovered a pathway for regulating absorption of fatty acids that could yield new targets for treating obesity. MFGE8, an integrin ligand, controls the uptake of fat in the gut and other tissues by co-opting a phosphoinositide 3-kinase signaling module better known for its involvement in insulin action. ${ }^{1}$ The team is working on inhibitors of the ligand or its receptors for use in obesity.

The findings provide a new way to stop fatty acids from getting into cells-a strategy for fighting obesity that has proved relatively intractable to date.

Roche's Xenical orlistat inhibits the lipase enzyme that releases fatty acids from dietary fats and thus decreases their absorption in the gut. However, although the compound has been marketed for obesity in the U.S. since 2007, it has limited efficacy and unpleasant side effects.

More recently, efforts have focused on the transporter proteins CD36 (GPIV) and solute carrier family 27 fatty acid transporter member

"The ability to regulate fatty acid absorption, through a divergent pathway that can also control glucose uptake, may provide a long-awaited opportunity in therapeutics focused on the direct inhibition of fatty acid uptake and possibly other nutrients." - Lex Van der Ploeg, Rhythm Pharmaceuticals Inc.

or with adding a mutant version of MFGE8 unable to bind to integrin receptors.

Conversely, Mfge 8 knockout in mice decreased fatty acid uptake in isolated adipocytes, liver cells, intestinal enterocytes and cardiac myocytes compared with wild-type $M f g e 8$ expression.

Furthermore, Mfge 8 knockout mice fed a high-fat diet did not develop obesity and were more sensitive to insulin than wild-type mice. The Mfge 8 knockout mice also had lower intracellular triglyceride levels than wild-type mice and had signs of reduced fatty acid transport.

Antibodies against the individual integrin $\alpha_{\mathrm{v}}$ and $\beta_{5}$ receptor subunits disrupted Mfge8 signaling and prevented rMfge8 from increasing uptake of fatty acid into adipocytes, enterocytes and hepatocytes.

The results provided a clear link between MFGE8 and fatty acid bsorption from the gut into the blood and various tissues.

The team then investigated the mechanism by which MFGE8 controls fatty acid uptake and found that the MFGE8 pathway converged on a phosphoinositide 3-kinase (PI3K)-based signaling module that is a known player in the insulin receptor pathway.

In addition to PI3K, the module includes protein kinase B (PKB; PKBA; AKT; AKT1) and $\mathrm{TBC} 1$ domain family member 4 (TBC1D4; AS160), a regulator of glucose transporter trafficking.

Activation of the pathway triggers translocation of CD36 and FATP1 from intracellular vesicles to the plasma membrane, in which the transporters mediate the uptake of fatty acids into the cytoplasm.

1 (SLC27A1; FATP1), which shuttle fatty acids into cells.

Arteria S.A. has a CD36 inhibitor in preclinical development for dyslipidemia.

Now, a team led by Kamran Atabai at UCSF has focused on the regulation of the transporters rather than on the proteins themselves and has found that MFGE8 (lactadherin; HMFG) acts as a master regulator of fatty acid uptake.

Atabai is an assistant professor in the UCSF Department of Medicine and a principal investigator at UCSF's Cardiovascular Research Institute and Lung Biology Center.

\section{Milking the fat connection}

Atabai's team identified MFGE8 in 2005. It is an integrin ligand that binds the integrin $\alpha_{v} \beta_{5}$ and integrin $\alpha_{v} \beta_{3}(C D 51 / C D 61)$ receptor complexes. ${ }^{2}$ Subsequent gene association and expression studies pointed to a connection between MFGE8 and obesity.,4

The researchers saw a connection between CD36 and MFGE8 because both showed overlapping functions in inflammatory pathways and were associated with obesity. As CD36 mediates fatty acid uptake into cells, they thought MFGE8 might act in concert with the transporter to control fat absorption.

In vitro, they found that adding recombinant MFGE8 (rMFGE8) to adipocytes increased fatty acid uptake compared with no treatment
Despite utilizing the same signaling module, MFGE8 and insulin produce different outcomes. Whereas MFGE8 causes translocation of fatty acid transporters to the cell surface, insulin uses the pathway to translocate a glucose transporter, solute carrier family 2 facilitated glucose transporter member 4 (SLC2A4; GLUT4), thus controlling glucose uptake.

Results were published in Nature Medicine.

Atabai is following up the study by looking for additional clinical evidence to support the MFGE8 connection with obesity. The team is prospectively collecting adipose tissue from patients with obesity in order to study MFGE8 levels and is performing a comprehensive genetic SNP analysis.

\section{Where MFGE8 meets insulin}

Lex Van der Ploeg, CSO of Rhythm Pharmaceuticals Inc., sees the MFGE8 findings as a promising step forward for obesity therapeutics.

"The ability to regulate fatty acid absorption, through a divergent pathway that can also control glucose uptake, may provide a longawaited opportunity in therapeutics focused on the direct inhibition of fatty acid uptake and possibly other nutrients," he told SciBX.

Rhythm's RM-493, a small peptide melanocortin 4 receptor (MC4R) agonist, is in Phase II testing for obesity. It is partnered with Ipsen Group. 
William Holland told SciBX that the connection between insulin and MFGE8 is likely to have an important impact on the field.

"It is amazing that the signaling machinery typically used by insulin-PI3K/AKT/AS160is harnessed by MFGE8 to regulate lipid uptake. How this is done in a context that minimally alters glucose uptake is remarkable," he said.

Because the goal is to limit lipid uptake, he added, it will be necessary to understand the process well to avoid exacerbating insulin signaling.

Holland is an assistant professor at The University of Texas Southwestern Medical Center and works on lipid signaling in metabolic disease.

According to Atabai, the increased insulin sensitivity in the high-fat diet-fed, Mfge 8 knockout mice suggests that blocking MFGE8 signaling could improve glucose control, too.

In obese individuals, he said, "MFGE8-induced fat absorption could induce insulin resistance, and inhibiting MFGE8-dependent fat absorption could enhance insulin sensitivity and ameliorate type 2 diabetes."

Thomas Hughes told SciBX, "The findings might point to new strategies to stimulate glucose transport by way of integrin inhibition. Such approaches could lead to a new class of insulin sensitizers."

Hughes is president and CEO of Zafgen Inc., which has beloranib in Phase II testing for the orphan obesity disease Prader-Willi syndrome. The compound is an inhibitor of methionine aminopeptidase 2 (MetAP2). Zafgen holds exclusive worldwide rights, outside Korea, for development and commercialization of beloranib. The company licensed the compound from Chong Kun Dang Pharmaceutical Corp.

However, according to Atabai, although his laboratory is developing antibodies against MFGE8, there might be unwanted effects of MFGE8 inhibition because of its involvement in other processes related to apoptosis and autoimmune functions.

Matthew Rodeheffer added that global MFGE8 inhibition could create problems by depriving other tissues of energy-delivering fatty acids. He suggested that the issue might be avoided by blocking MFGE8 locally in adipose tissue to prevent effects in other cells such as cardiomyocytes.

An alternative, he said, would be to block MFGE8 locally in the gut, through which fatty acids initially gain access to the body via enterocytes in the gut lining.

Rodeheffer is an assistant professor of comparative medicine and of molecular, cellular and developmental biology at Yale University. $\mathrm{He}$ is an expert on adipocyte development.

Holland was cautious about a local treatment in the gut.

"Many lipids, particularly long-chain, saturated fatty acids, are very proinflammatory in the gut. Strong and chronic MFGE8 inhibition and concomitant elevation of these species therefore could have unwanted consequences," he told SciBX.

Atabai told SciBX that he thinks blocking the pathway via integrin receptors might produce a better safety profile. His team will focus on local enteric and global integrin $\alpha_{v} \beta_{5}$ blockade because it is safer in animals than integrin $\alpha_{\mathrm{v}} \beta_{3}$ inhibition, which produces diverse effects.

UCSF has filed a provisional patent on the findings. The IP is available for licensing.

Boettner, B. SciBX 7(6); doi:10.1038/scibx.2014.159

Published online Feb. 13, 2014

\section{REFERENCES}

1. Khalifeh-Soltani, A. et al. Nat. Med.; published online Jan. 19, 2014; doi:10.1038/nm.3450

Contact: Kamran Atabai, University of California, San Francisco, Calif. e-mail: kamran.atabai@ucsf.edu

2. Atabai, K. et al. Mol. Biol. Cell 16, 5528-5537 (2005)

3. Henegar, C. et al. Genome Biol. 9, R14; published online Jan. 21, 2008; doi:10.1186/gb-2008-9-1-r14

4. Rankinen, T. et al. Obesity (Silver Spring) 14, 529-644 (2006)

COMPANIES AND INSTITUTIONS MENTIONED

Arteria S.A., Caissargues, France

Chong Kun Dang Pharmaceutical Corp. (KSE:001630), Seoul, South Korea

Ipsen Group (Euronext:IPN; Pink:IPSEY), Boulogne-Billancourt, France

Rhythm Pharmaceuticals Inc., Boston, Mass.

Roche (SIX:ROG; OTCQX:RHHBY), Basel, Switzerland

University of California, San Francisco, Calif.

The University of Texas Southwestern Medical Center, Dallas, Texas

Yale University, New Haven, Conn.

Zafgen Inc., Cambridge, Mass. 